\title{
Les ailes de la centralité, réseaux aériens planétaires et mondialisation
}

Wings of centrality, planetary air networks and globalization

Las alas de la centralidad: redes aéreas plantearias y mundialización

\section{Hervé Théry}

\section{(2) OpenEdition}

\section{Journals}

Édition électronique

URL : http://journals.openedition.org/mappemonde/2044

DOI : 10.4000/mappemonde.2044

ISSN : $1769-7298$

Éditeur

UMR ESPACE

Référence électronique

Hervé Théry, «Les ailes de la centralité, réseaux aériens planétaires et mondialisation », Mappemonde [En ligne], 119 | 2017, mis en ligne le 01 janvier 2017, consulté le 02 juillet 2020. URL : http://

journals.openedition.org/mappemonde/2044 ; DOI : https://doi.org/10.4000/mappemonde.2044

Ce document a été généré automatiquement le 2 juillet 2020

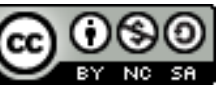

La revue Mappemonde est mise à disposition selon les termes de la Licence Creative Commons Attribution - Pas d'Utilisation Commerciale - Partage dans les Mêmes Conditions 4.0 International. 


\title{
Les ailes de la centralité, réseaux aériens planétaires et mondialisation
}

\author{
Wings of centrality, planetary air networks and globalization \\ Las alas de la centralidad : redes aéreas plantearias y mundialización
}

\section{Hervé Théry}

1 Dans un monde globalisé, quoi de plus globalisé que le transport aérien, dont l'espace de référence est la planète, et qui relie des villes distantes de centaines - voire de milliers - de kilomètres «à vol d'oiseau », en survolant les frontières nationales ? Cela ne veut pas dire qu'il n'ait pas sa géographie, faite de points-clés - les aéroports plus ou moins fréquentés - et ses lignes de force, les bien nommées lignes aériennes. Celles-ci sont inégalement parcourues par des acteurs en forte compétition entre eux, les compagnies aériennes, de plus en plus associées en alliances qui rivalisent pour dominer le monde.

Or, il existe une source facilement accessible pour repérer ces points et avoir une idée de ces flux, grâce à l'exploitation des données des transpondeurs des avions en vol $^{1}$ et aux cartes que les compagnies publient largement, évidemment pas pour le plaisir des géographes mais dans l'espoir de capter des passagers souhaitant se rendre dans telle ou telle ville. Pour se procurer ces cartes, il n'est plus nécessaire de prendre leurs avions pour accéder aux revues de bord, où elles figurent toujours dans les dernières pages, et de les arracher subrepticement ${ }^{2}$. On les trouve désormais, pour la plupart d'entre elles, sur le site des compagnies, plus ou moins réussies, plus ou moins belles, mais en tout cas suFfisantes pour se faire une idée de leurs stratégies, qui s'inscrivent dans le choix des lignes qu'elles choisissent de desservir.

3 Commençant par le niveau global, toutes compagnies confondues, on passera ensuite à celui des alliances et des compagnies, en concurrence entre elles, et l'on verra enfin les efFets d'un mouvement de conquête des réseaux mondiaux par certains pays 
émergents, ce qui change le centre de gravité des réseaux, et mettant - selon certains le Moyen-Orient et l'hémisphère du Pacifique au centre du monde.

On peut ainsi présenter une vision synthétique de ces réseaux planétaires à partir d'une source, accessible et constamment renouvelée, sans autre ambition que de tirer de ces cartes publiées par les compagnies, par une analyse critique, ce qu'elles peuvent nous dire et ce que nous pouvons en dire. Pour approfondir cette analyse il faudrait ensuite passer à l'échelle nationale - surtout pour les grands pays - et disposer de données sur les flux qui parcourent ces lignes, ainsi que sur les coûts d'accès, ce qui est esquissé dans le cas du Brésil à la fin de l'article. L'ambition de cet article est donc modeste, bien qu'il embrasse le monde, pour aller plus loin on devra se reporter aux travaux menés par des collègues spécialisés, notamment J. Varlet (1997), P. Biplan (2004), N. Cattan (2004), M. Amiel, G. Melançon et C. Rozenblat (2005), M. Strale (2013) cités en bibliographie.

\section{Réseaux planétaires}

Une remarquable représentation des réseaux aériens mondiaux est disponible sur le site OpenFlights, the site for flight logging, mapping, stats and sharing. Y figure une série de cartes des vols longue-distance et intercontinentaux. Reprise sur Wikipédia en 16 langues, elle montre selon la version française de celui-ci «le caractère "nodal" et arborescent du réseau mondial de transport aérien, et sa densité en Europe et aux États-Unis et plus généralement sur l'hémisphère nord ».

6 De fait, on y voit bien la densité des réseaux dans et entre les mégalopoles nordaméricaine, européenne et asiatique, des «couloirs» vers l'Amérique du Sud et l'Australie, et la faiblesse des réseaux en et vers l'Afrique. Dans le détail on pourrait détecter, en fonction des conjonctures politiques et militaires, que certaines régions du monde sont évitées (Ukraine, notamment dans sa partie orientale, Irak et Syrie, etc.), ce qui rend délicate actuellement la liaison entre l'Europe d'une part, le Golfe et l'Extrême-Orient de l'autre.

Openflights y ajoute une dimension médicale : «ce réseau est notamment suivi par les épidémiologistes, car susceptible de rapidement véhiculer des maladies pandémiques (un éventuel virus grippal hautement pathogène et très contagieux, ou SRAS, par exemple). Les grands aéroports sont maintenant intégrés dans les systèmes de veille et d'alertes sanitaires mis en place sous l'égide de l'OMS avec l'ONU » (Cf. cet article de la Wikipedia).

8 La première version de la carte était dans une projection classique (Mercator probablement) mais comme l'expliquent les auteurs, ils ont ensuite entrepris de faire mieux, pour rendre compte de la rotondité de la terre. Le premier essai en ce sens ( figure 1), comme ils le racontent (en traduction libre et abrégée), «semblait un peu étrange, parce qu'il montrait le monde entier écrasé sur un disque, y compris les parties que l'on ne pourrait pas voir réellement si l'on était dans l'espace et regardait la Terre ». Ils précisent même "pour les nerds en cartographie, c'est une projection équivalente Lambert azimutale. La seule façon d'y arriver est d'étirer les morceaux sur les bords, ce qui explique pourquoi la pauvre Australie semble si étrange ».

Encouragés par cette première amélioration, ils ont continué : «Notre deuxième tentative a utilisé une projection orthographique, assez proche de ce qu'on pourrait 
réellement voir de l'espace. Le seul problème est qu'on ne peut voir que la moitié du monde, et donc seulement la moitié des routes aériennes. La solution a été d'ajouter le temps dans l'équation, et de transformer la carte en une vidéo ». Celle-ci est accessible à l'adresse http://vimeo.com/12078596, avec une version à plus basse définition pour les utilisateurs ne disposant pas d'une bonne connexion à l'adresse http://vimeo.com/ 12077446.

Figure 1. Les grandes routes aériennes mondiales

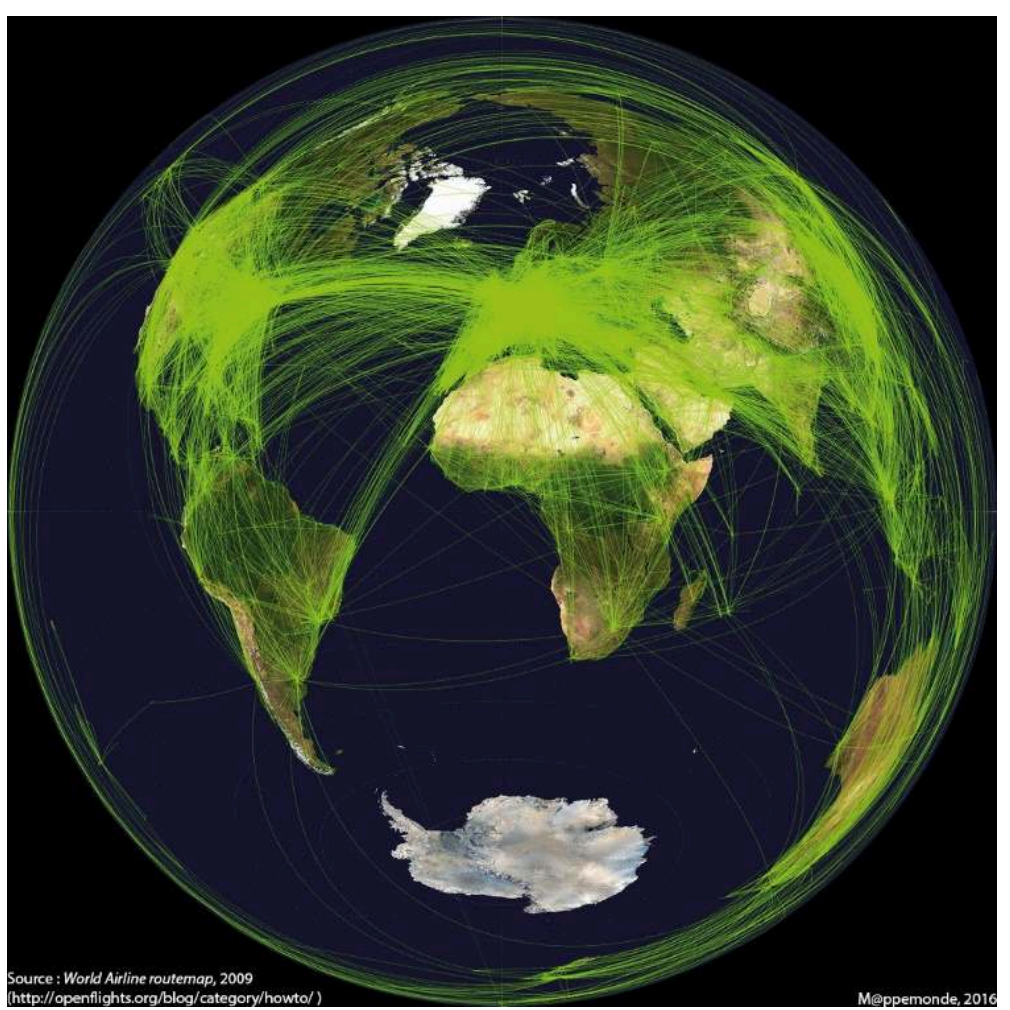

Source : World Airline routemap, 2009

https://blog.openflights.org/category/howto

D'autres sites permettent d'avoir une vision dynamique des flux aériens soit par une animation qui exploite le suivi des transpondeurs des avions en $\mathrm{vol}^{3}$, soit en localisant, à un moment donné, les vols en cours. Parmi les premiers un petit film condensant 24 heures de circulation aérienne dans le monde est disponible sur YouTube à plusieurs adresses ${ }^{4}$. Il montre non seulement la concentration géographique des couloirs aériens les plus fréquentés mais aussi la concentration de vols dans le temps, et l'on y voit littéralement les pays se réveiller et s'endormir, les uns après les autres. Graphiquement plus recherchée, une autre animation est diffusée sur le site du quotidien britannique The Guardian. (figure 2) 
Figure 2. «Plane in flight »

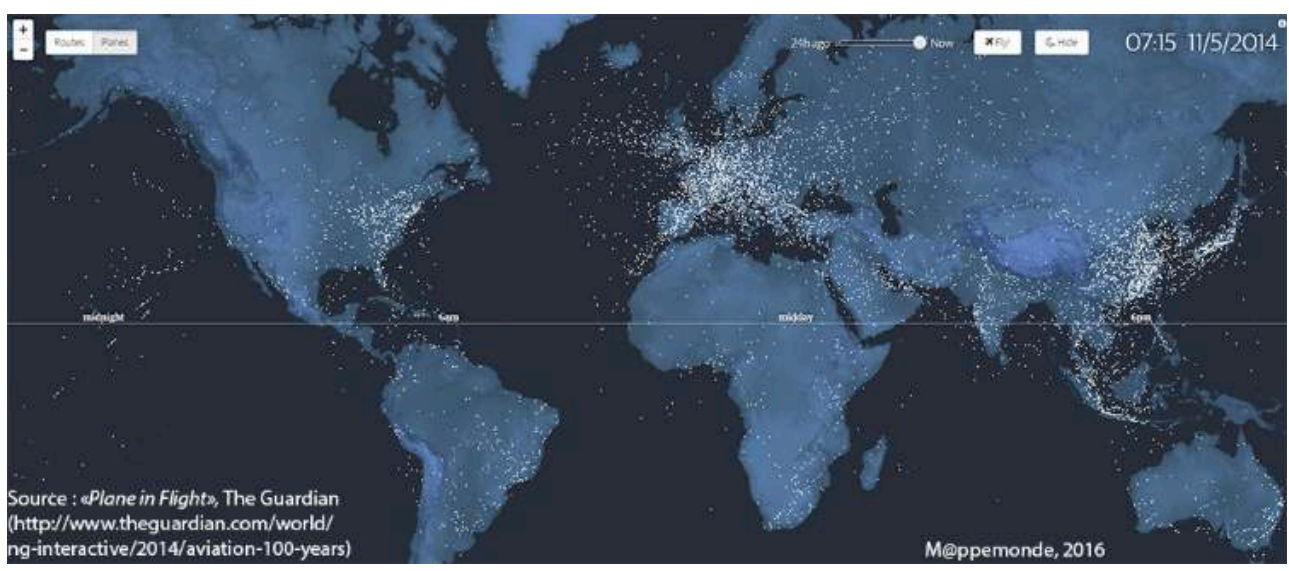

Source : "Plane in Flight », The Gardian

https://www.theguardian.com/world/ng-interactive/2014/aviation-100-years

Parmi les sites localisant les avions en vol on peut en citer deux, qui difFèrent légèrement par les choix graphiques, Flightradar 24 (figure 3) et Planefinder. Outre leur utilité principale - rassurer les proches des voyageurs en leur permettant de suivre minute par minute l'avion dans lequel voyage l'être cher-ils montrent bien l'accumulation des vols sur certaines zones du monde et le vide de certaines autres comme le Grand Nord et l'Afrique.

Figure 3. Flightradar 24, situation le 9/08/2015 à 15h23 UTC

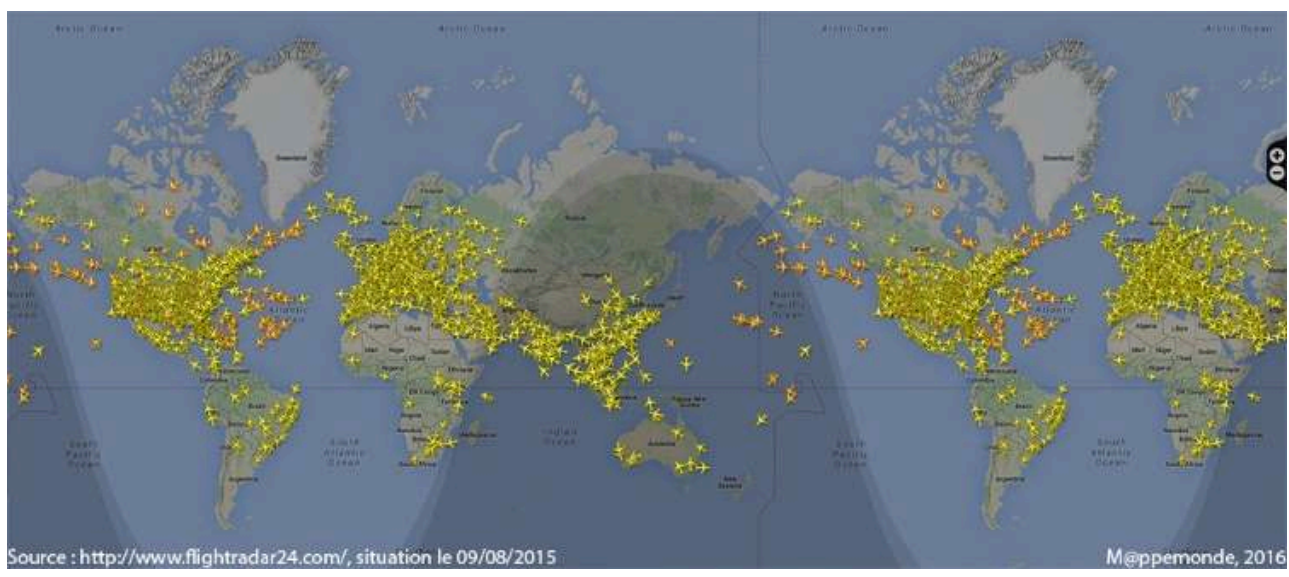

Source : http://www.flightradar24.com

\section{Trois grandes alliances}

12 Passant de l'échelle globale d'un monde (graphiquement unifié, mais déjà marqué par des inégalités bien visibles), à celui des acteurs économiques en compétition, on rencontre les grandes alliances de compagnies aériennes, dont même le plus distrait des voyageurs ne peut ignorer le nom, tant il est martelé dans les annonces faites à bord par le personnel navigant.

Les avantages pour les compagnies à entrer dans ces alliances sont multiples et, à part les compagnies low-cost (qui ont d'autres stratégies, avec des vols de point à point, sans 
hub ni possibilité de correspondance) et les compagnies classées comme dangereuses (qui en sont exclues) $)^{5}$, la plupart d'entre elles se rallient progressivement à ces trois alliances, avec parfois des changements d'allégeances liées aux fusions, nombreuses dans ce secteur au cours des dernières décennies.

Selon Mathieu Strale l'offre des alliances représentait, à la date où il écrivait son article (publié en 2013), environ la moitié du total de l'ofFre aérienne régulière mondiale : « concentrées dans les pays du centre, où leur part de trafic est supérieure à $40 \%$, les alliances commencent à s'étendre vers les pays de la périphérie les plus avancés ». En revanche "dans les régions mondiales les plus périphériques, l'Afrique et l'Asie centrale, aucune compagnie n'est membre d'une alliance, ce qui est lié aux conditions d'accès dans les alliances : il faut répondre à des conditions financières, de qualité et de sécurité que n'atteignent pas toujours les compagnies d'Afrique et d'Asie. De plus, le faible trafic aérien à l'intérieur de ces régions rend peu intéressant l'installation d'un $h u b$, et le trafic depuis le reste du monde peut être assuré depuis les grands hubs européens et d'Asie du Sud-Est ».

Il y a là une logique forte et globale, car comme l'indique le site Voyager en avion, «l'objectif de ces alliances est de renforcer la coopération entre compagnies afin d'offrir aux voyageurs un vaste réseau de destinations dans le monde entier. Pour augmenter la fluidité des déplacements des voyageurs, les compagnies d'une même alliance font en sorte de faciliter les correspondances, notamment dans les hubs. Les alliances proposent aussi des programmes de fidélisation permettant à leurs clients de favoriser les compagnies adhérentes [...] La première date de 1989, lorsque Northwest Airlines et KLM ont élaboré pour la première fois un partenariat favorisant le partage de code à grande échelle. Quelques années plus tard, en 2000, ces deux mêmes compagnies fondaient SkyTeam avec Air France et Delta Airlines [...]. Aujourd'hui, trois alliances se livrent une guerre sans merci, Star Alliance est en tête et Oneworld peine à rattraper ses concurrentes [...]

- Star Alliance, basée à Francfort-sur-le-Main en Allemagne, compte 27 compagnies membres autour de la Lufthansa et d'United, avec un total d'environ 4380 appareils. L'alliance dessert 193 pays grâce aux 1356 aéroports sur lesquels elle se pose. Chaque jour, ce sont plus de 21500 vols qui sont assurés par les compagnies membres de Star Alliance pour près de 680 millions de passagers transportés chaque année. [...]

- SkyTeam, inaugurée en 2000 et basée aux Pays-Bas à Haarlemmermeer, est la deuxième, parmi ses 19 membres, elle compte Air France et KLM. L'alliance dispose de 4050 avions lui permettant d'assurer 15000 vols quotidiens. Chaque année, elle transporte 550 millions de voyageurs vers 187 pays.

- Troisième sur le podium, Oneworld est ambitieuse et souhaite détrôner ses concurrentes. Grâce à la fusion entre American Airlines et US Airways, elle détient désormais la plus grande compagnie aérienne mondiale. Créée en 2000 et située à New York, elle compte 13 membres [et] une flotte de près de 2500 appareils. Elle efFectue quotidiennement 9360 vols entre 870 aéroports de 146 pays. Chaque année, elle transporte 335 millions de voyageurs ».

16 À en juger par les cartes des aéroports qu'elles desservaient en 2012 et des liaisons qu'elles assuraient alors (figure 4), les trois alliances ont sensiblement les mêmes stratégies de couverture de l'espace mondial, avec des points forts en Europe, en Amérique du Nord et en Asie, et peu de choses ailleurs. Tout au plus peut-on constater que OneWorld couvre un peu mieux le Pacifique Sud et Star Alliance l'Atlantique Sud et l'Afrique. 
Figure 4. Trois alliances mondiales, situation en 2012

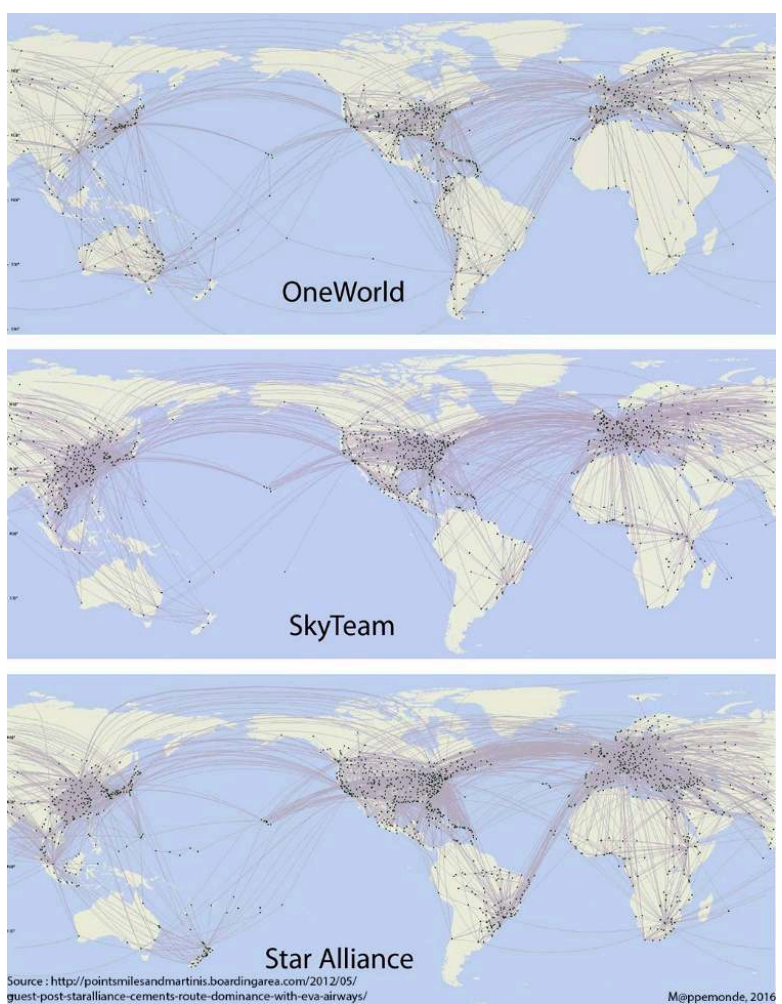

Source : https://pointsmilesandmartinis.boardingarea.com/2012/05/guest-post-staralliance-cementsroute-dominance-with-eva-airways

\section{Les réseaux des compagnies}

17 Dans les alliances cohabitent des compagnies, qui gardent leurs spécificités. Le travail de James Cheshire, professeur au Département de géographie de l'University College London, qui fait partie de son blog spatial.ly, confronte les configurations spatiales des plus grandes compagnies aériennes mondiales (figure 5). 
Figure 5. Les réseaux des principales compagnies mondiales

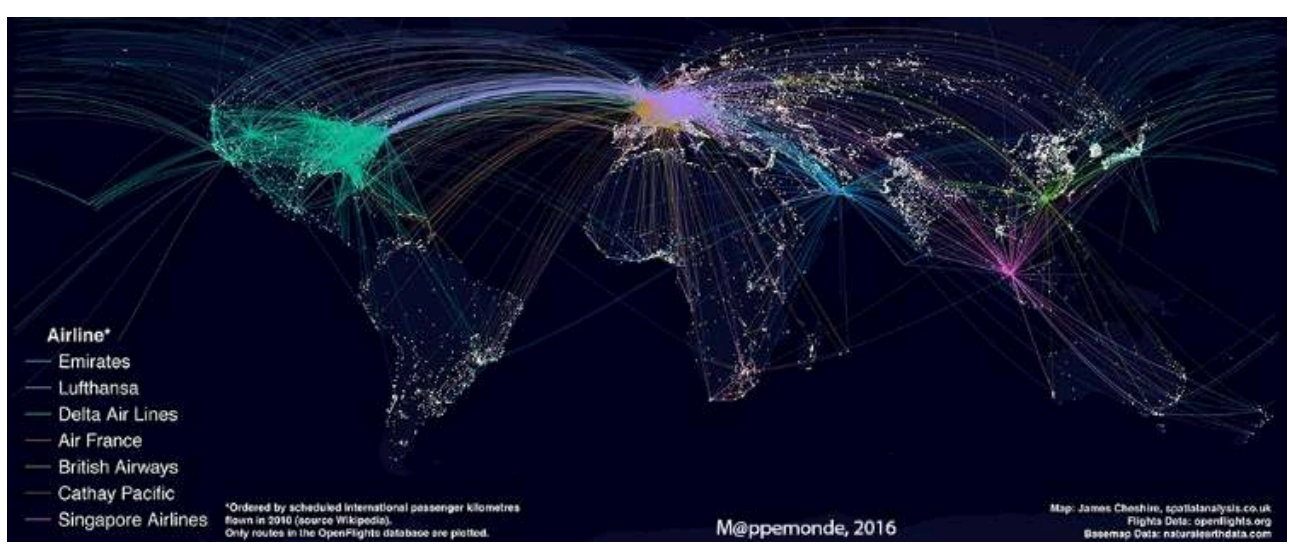

Ordered by scheluded international passengers kilometres flown in 2010 (source wikipedia). Only routes in the Openflights database are plotted.

Source: http://spatial.ly/2012/06/mapping-worlds-biggest-airlines

Map: James Cheshire, http://spatialanalysis.co.uk

Flights data: https://openflights.org

Basemap data: http://naturalearthdata.com

18 La carte qu'il a produite "montre les routes parcourues par les sept premières compagnies aériennes (selon le critère de la distance parcourue par leurs passagers). Le fond de carte montre les grandes zones urbaines et j'ai essayé de le faire ressembler un peu à la belle image composite Earth at Night produite par la NASA. On y voit clairement une relation entre les lieux où les gens vivent et ceux vers où les grands transporteurs volent, en Europe et aux États-Unis, mais l'Inde et une bonne part de la Chine présentent relativement peu de routes. Je suppose que la relève est assurée par les petites compagnies aériennes dans ces pays [qui] doivent représenter les secteurs de croissance de l'économie mondiale, de plus en plus entraînée par l'Est ».

Peut-être aurait-il été plus pertinent, s'agissant de géométrie des réseaux, de retenir plutôt les compagnies desservant le plus grand nombre de destinations (tableau 1), mais sans doute le résultat aurait-il été peu différent, et la stratégie des alliances vise précisément à associer des entreprises qui ont leurs points forts dans différentes parties du monde, de façon à créer des complémentarités.

Tableau 1. Les dix premières compagnies mondiales par nombre de destinations

\begin{tabular}{|l|l|}
\hline Compagnie & Destinations \\
\hline 1. United Airlines & 369 \\
\hline 2. American Airlines & 340 \\
\hline 3. Delta Air Lines & 333 \\
\hline 4. Turkish Airlines & 254 \\
\hline 5. Lufthansa & 218 \\
\hline 6. China Eastern Airlines & 211 \\
\hline
\end{tabular}




\begin{tabular}{|l|l|}
\hline 7. British Airways & 197 \\
\hline 8. Air France & 194 \\
\hline 9. China Southern Airlines & 193 \\
\hline 10. Air Canada & 181 \\
\hline
\end{tabular}

Source : https://en.wikipedia.org/wiki/Largest_airlines_in_the_world plus petites, qui leur ouvrent l'accès à d'autres parties du monde. On peut s'en convaincre en comparant les dessertes des compagnies qui forment la Star Alliance, le groupe qui en rassemble le plus grand nombre (27) et dessert le plus grand nombre de pays (193), pratiquement le monde entier.

21 Classées par ordre alphabétique sur la figure 6, elles sont clairement de plusieurs types, même en ne retenant que celle qui couvrent plusieurs continents et donc en excluant celles qui n'ont qu'une desserte régionale (Adria, Aegean, Croacia Airlines, Lot Polish Airlines et Shenzen Airlines).

Figure 6. Les réseaux des compagnies de la Star Alliance

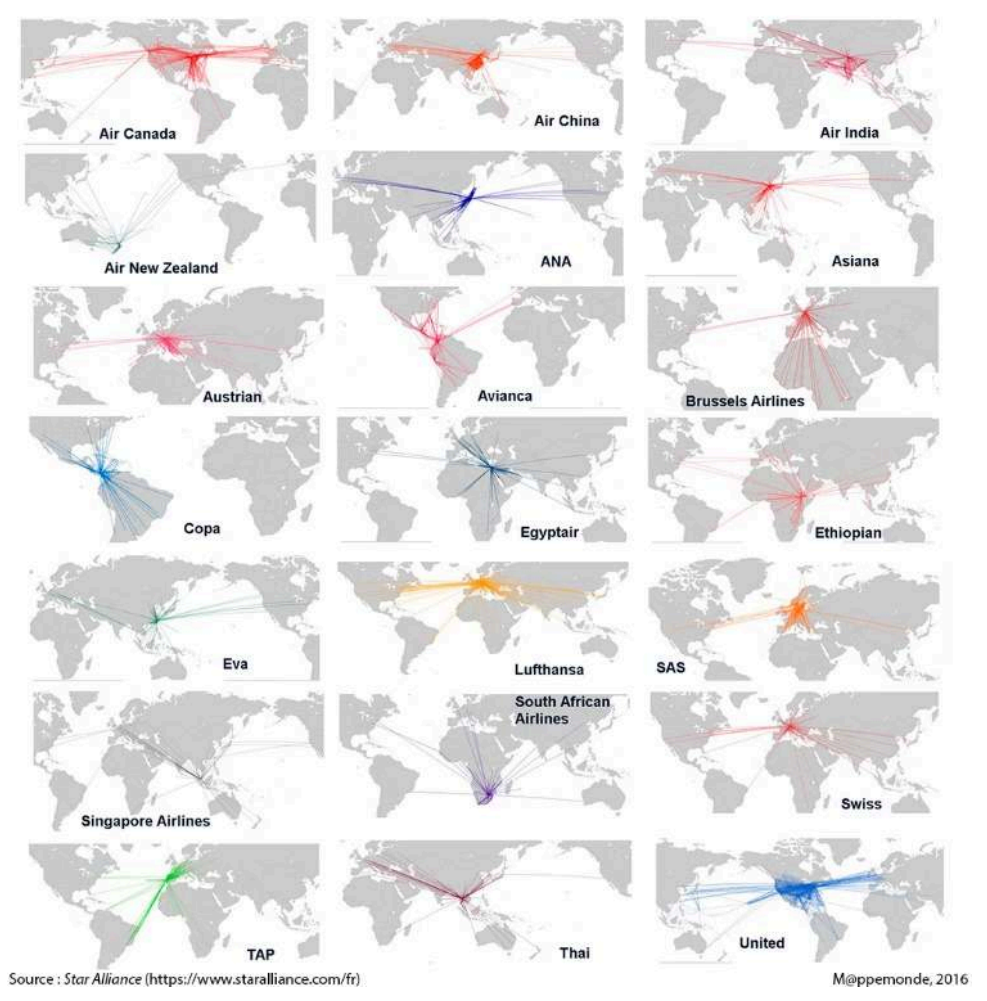

Source : Star alliance, https://www.staralliance.com

Des constantes apparaissent clairement (figure 7): toutes ont des liaisons avec l'Amérique du Nord, le principal marché mondial. Celles qui sont basées en Asie desservent l'Europe, et vice-versa, ce qui «ferme » le principal triangle mondial. Les deux principales asiatiques du groupe vont en Océanie, et la seule océanienne va en 
Asie, en Europe et en Amérique du Nord. Les trois africaines vont partout, mais seules deux européennes desservent l'Afrique. Les deux sud-américaines se cantonnent à l'Amérique du Nord et à l'Europe.

Figure 7. Schéma des échanges dans la Star Alliance
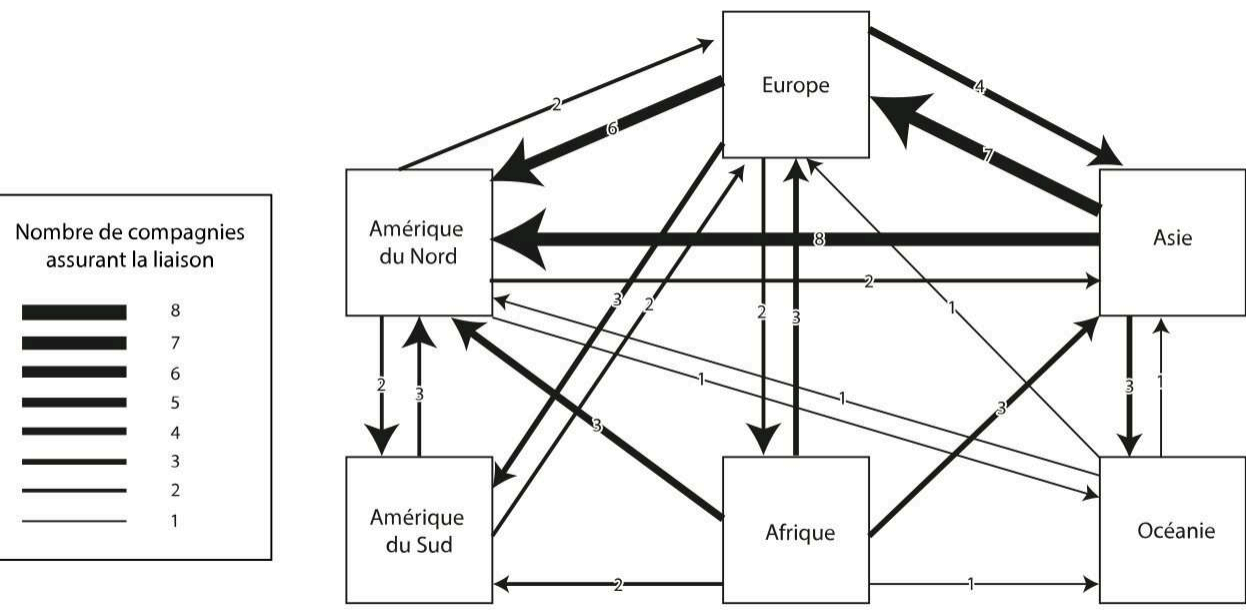

Conception \& réalisation : Hervé Théry - 2016 - Cartes et Données

Conception et réalisation : Hervé Théry - 2016 - Cartes et Données

Le réseau de chaque compagnie a en effet sa topologie particulière, qui dépend des relations que chacune, à partir de sa base principale, entretient avec le reste du monde. Les héritages historiques pèsent évidemment, par les liaisons entre pays unis par une même langue et un passé colonial, comme entre le Portugal et le Brésil pour la TAP6. Mais ce sont bien sûr les volumes des échanges migratoires, économiques et touristiques qui sont déterminants, car chaque ligne, même si sa création est parfois décidée de façon un peu volontariste, pour des raisons souvent diplomatico-politiques, ne survit que si un nombre suFfisant de passagers payants la fréquentent et en assurent la rentabilité.

En observant ces cartes, et celles que l'on trouve sur leurs sites, on remarque pourtant que certaines compagnies ont un réseau d'une topologie très particulière, en raison de leur histoire propre et de la genèse des réseaux internationaux, un privilège qui est toutefois aujourd'hui remis en cause par des nouveaux venus, aux vastes ambitions.

\section{Puissances coloniales, pays émergents et Pacifique}

Les principales anciennes puissances coloniales, France et Grande-Bretagne, sont aussi les pays où l'aviation commerciale a débuté, à partir desquels des réseaux ont commencé à se développer vers l'Afrique et vers l'Asie, alors largement sous leur contrôle. Vers les Amériques, les routes de l'Atlantique nord ont commencé à fonctionner dès que la technique l'a permis, d'abord avec des escales techniques (en Irlande et à Terre-Neuve), puis d'un coup d'aile, tout comme celles de l'Atlantique sud, avec puis sans escales africaines. Il est donc facile de comprendre que les liaisons entre les Amériques, d'une part, et l'Asie, l'Océanie et l'Afrique, d'autre part, se soient longtemps faites via l'Europe, qui bénéficiait littéralement d'une rente de situation tant 
que les avions ne pouvaient facilement franchir les immensités vides du Pacifique ou le continent africain, faute de relais où se poser et se ravitailler.

C'est à ces héritages que les compagnies européennes, principalement Air France et British Airways, doivent de disposer aujourd'hui encore de réseaux couvrant le monde entier (figure 8), une impression renforcée par le choix de la projection cartographique qui met l'Europe au centre du planisphère et place la coupure (nécessaire pour « dérouler » la sphère terrestre sur le plan de la carte) dans le détroit de Béring.

Figure 8. Les anciennes puissances coloniales au sommet du monde
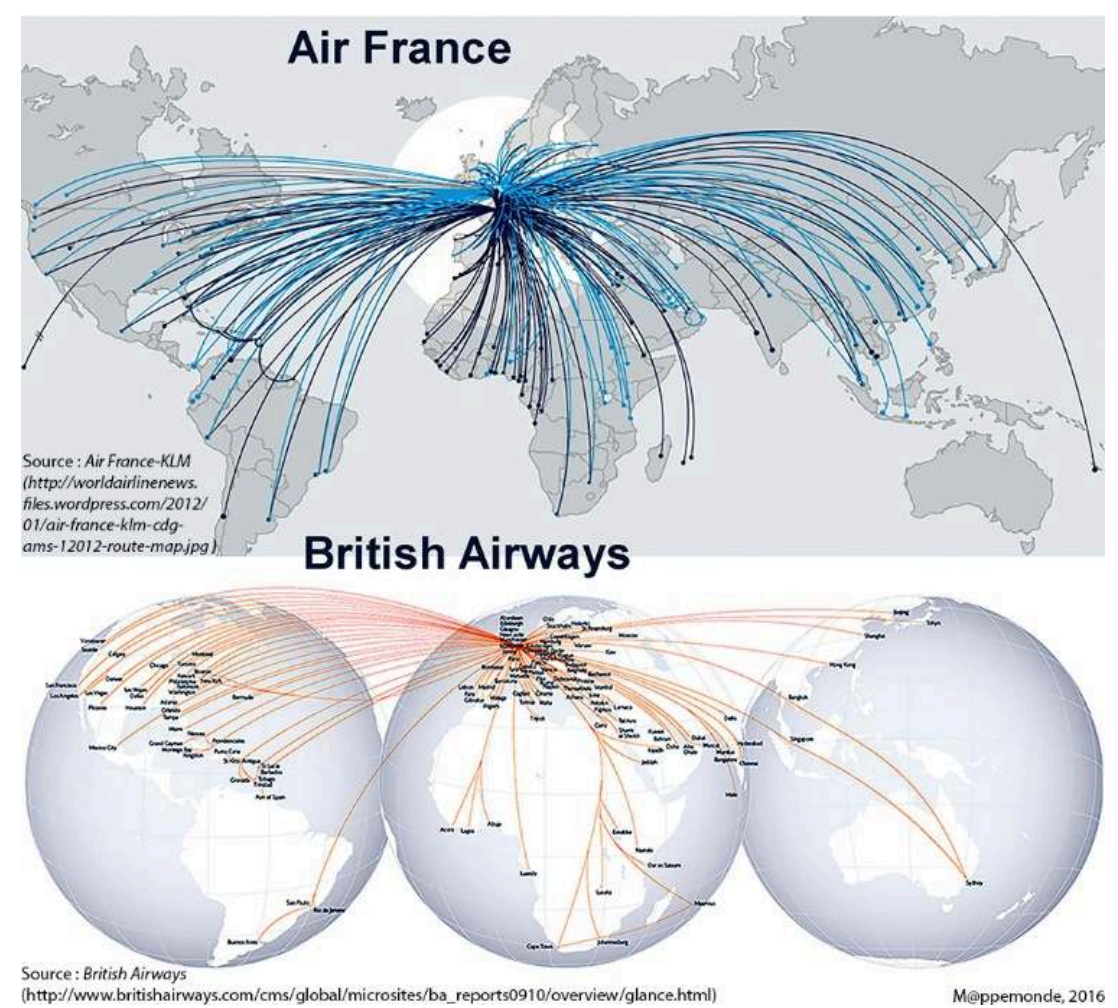

Sources : Air France-KLM, https://worldairlinenews.files.wordpress.com/2012/01/air-france-klm-cdgams-12012-route-map.jpg ; British Airways, https://www.britishairways.com/cms/global/microsites/ ba-reports0910/overview/glance.html

Cet européocentrisme est aujourd'hui contesté par des pays émergents qui s'appuient sur leur nouvelle puissance économique et sur les progrès de l'aviation pour tenter de détourner à leur profit les flux mondiaux.

Un des plus remarquables de cette tendance est le développement des hubs des Émirats Arabes Unis, au premier rang desquels celui de Dubaï, dont l'ambition est telle qu'il a choisi de donner à son nouvel aéroport, Al Maktoum International Airport, le nom modeste - de Dubaï World Central, Dubaï centre du monde (figure 9). 
Figure 9. Dubai World Central
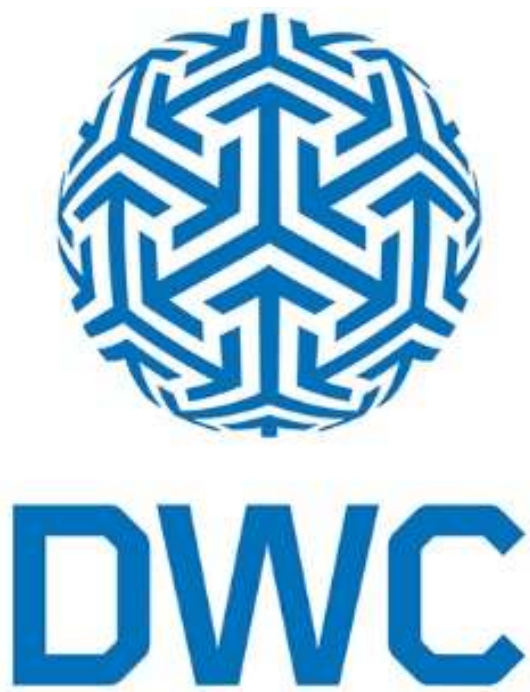

\section{DUBAI WORLD CENTRAL

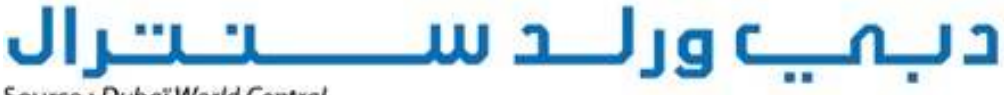 \\ Source : Dubaï World Central \\ (http://www.uaefreezones.com/fz_dubai_logistics_city.html \\ M@ppemonde, 2016}

Source : Dubaï World central, http://www.uaefreezones.com/fz_dubai_logistics_city.html

Au-delà de la rhétorique, cette ambition a une base réelle. D'une part, les compagnies du Golfe ont commencé à capter les passagers qui souhaitent se rendre directement des Amériques au Moyen-Orient et surtout en Asie, évitant ainsi le transit par l'Europe, raccourcissant considérablement le trajet (figure 10). D'autre part, le projet de construction du nouvel aéroport (pour remplacer celui du centre-ville, figure 11) s'accompagne d'un vaste projet urbain, fondé sur l'accès aérien. 
Figure 10. Dubaï au milieu du monde

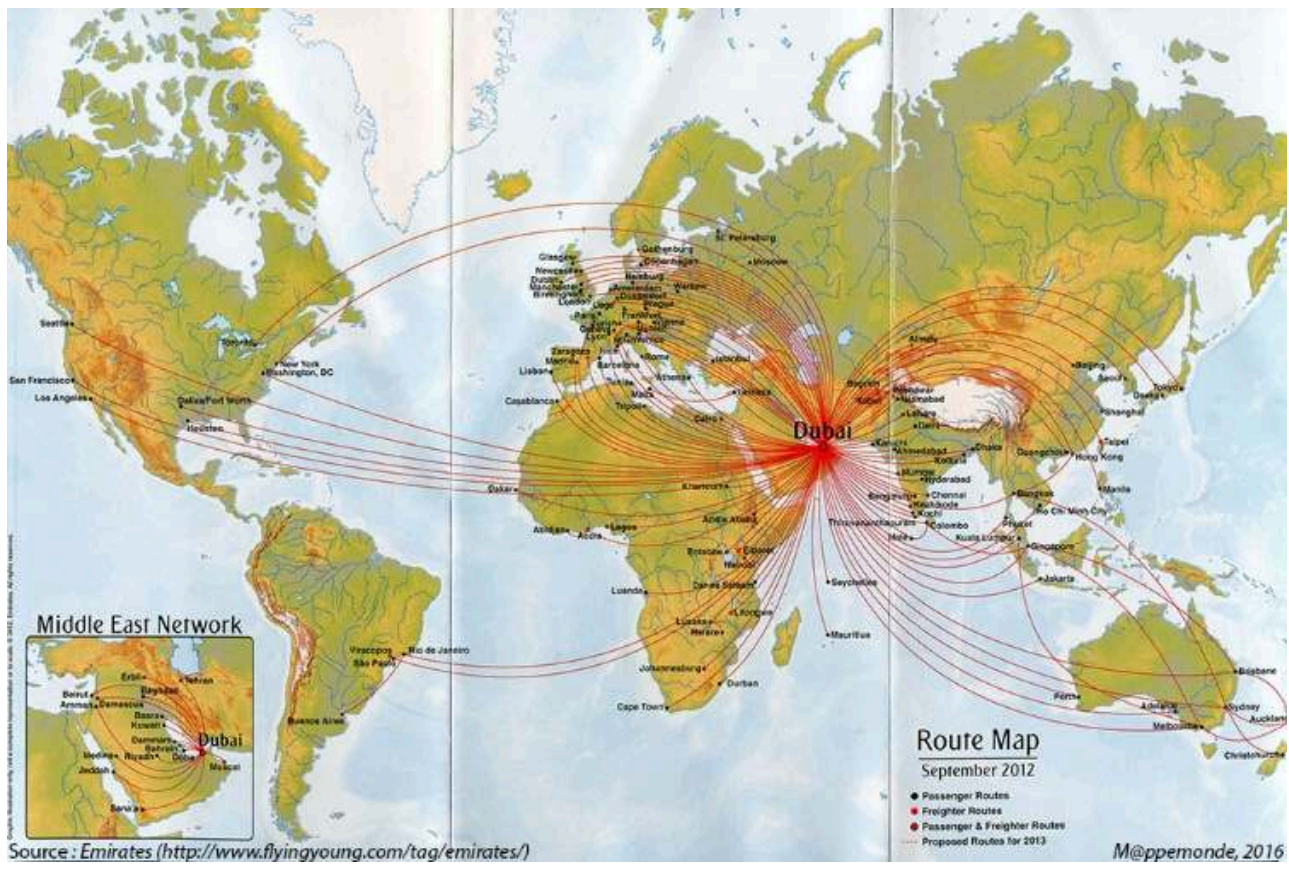

Source : Emirates, http://www.flyingyoung.com/tag/emirates

Selon le site de l'aéroport Dubai World Central (DWC), "Aujourd'hui le monde des afFaires exige des vitesses de livraison de plus en plus rapides et des niveaux plus élevés de connectivité. Cela fait grimper la demande pour le transport aérien, au point que $35 \%$ du commerce mondial en valeur en dépend maintenant - un pourcentage qui va sûrement augmenter au fil du temps. Aujourd'hui, un aéroport est un catalyseur économique et a élargi son influence au-delà des frontières traditionnelles pour englober un développement urbain centré autour de lui, formant une « aérotropole ». Se fondant sur [ce] modèle Dubai World Central (DWC) est le résultat d'un plan directeur conçu avec soin, qui définit le cadre pour l'avenir économique de Dubaï. Une superficie de 140 kilomètres carrés a été définie pour créer une zone économique [comprenant] une multitude d'activités, y compris la logistique, l'aviation, commerciale, des expositions, des entreprises humanitaires, résidentielles et de loisirs autour d'un aéroport de classe mondiale, avec la capacité annuelle prévue de 12 millions de tonnes de marchandises et de 160 millions de passagers ». 
Figure 11. Dubaï et ses deux aéroports

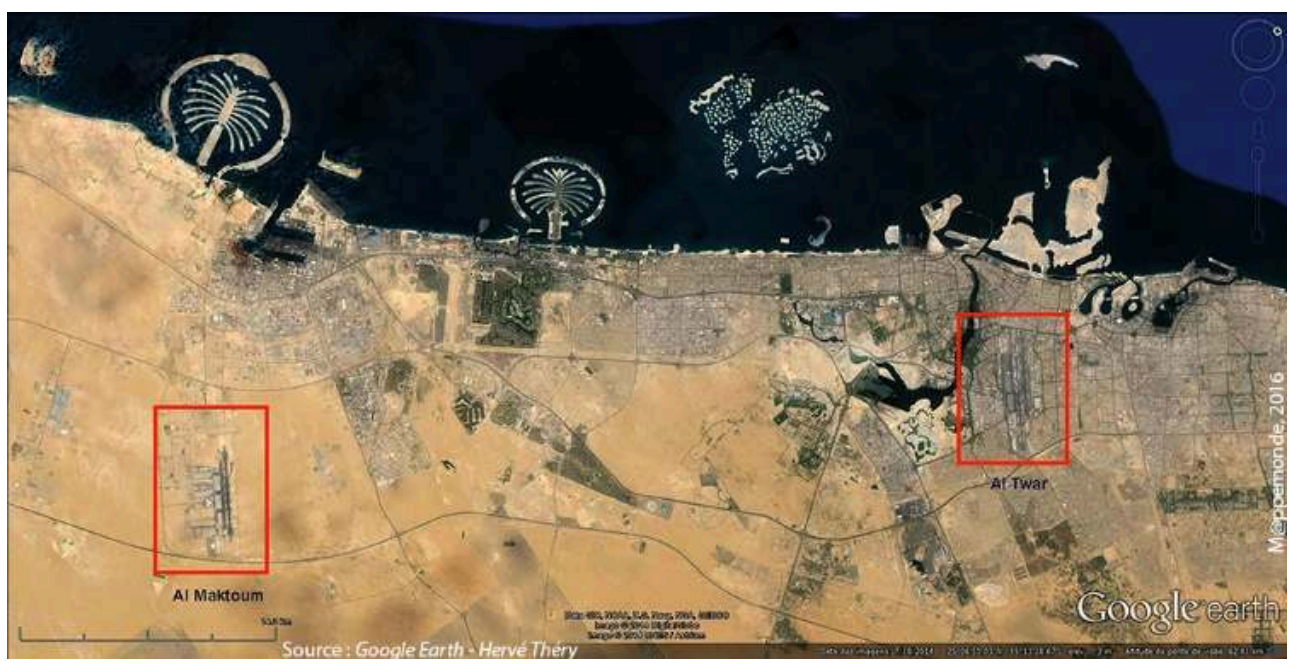

Source : Google Earth - Hervé Théry

31 Un autre cas remarquable de nouvelle centralité est celui de la Chine, dont la montée en puissance économique et diplomatique s'accompagne d'une croissance de la demande de vols depuis et vers les aéroports chinois. Les cartes de routes aériennes publiées par les compagnies chinoises reflètent bien les orientations majeures de ces vols, vers l'Europe et vers les Amériques, et adoptent des projections qui situent le pays à ce qui est pour les habitants de l'Empire du Milieu sa juste place, au centre. De ce point de vue, Chinois de Taiwan et du continent sont pour une fois d'accord (figure 12).

Figure 12. Les liaisons aériennes de la China Eastern Airlines

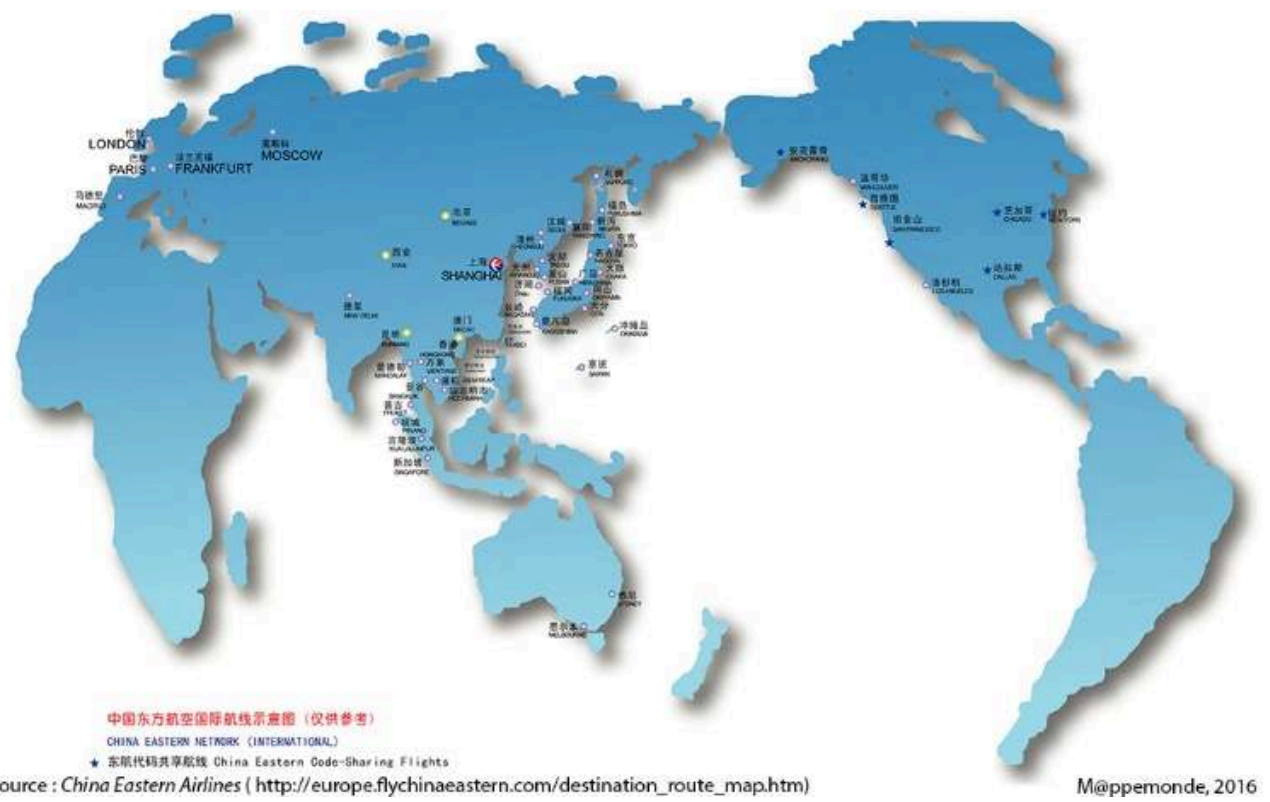

Source : China Eastern Airlines, http://europe.flychinaeastern.com/destination_route_map.htm

32 De ce fait, on voit se dessiner une nouvelle situation du Pacifique, qui refait son apparition sur les cartes au lieu d'être sacrifié par les projections centrées sur l'Eurasie, et les compagnies qui y ont leur base principale bénéficient à leur tour d'une rente de situation entre Amériques, Asie et Océanie, les Hawaiian Airlines, par exemple ( 
figure 13). Cela n'en fait pas pour autant le centre du monde, n'en déplaise à certaines compagnies qui en font un des axes majeurs de leur communication. Si cela peut être crédible en ce qui concerne Dubaï et la Chine, cela relève quand même d'une anticipation audacieuse dans le cas du Pacifique, comme le montre le vide aérien des cartes des flux mondiaux.

Figure 13. Le Pacifique, centre du monde

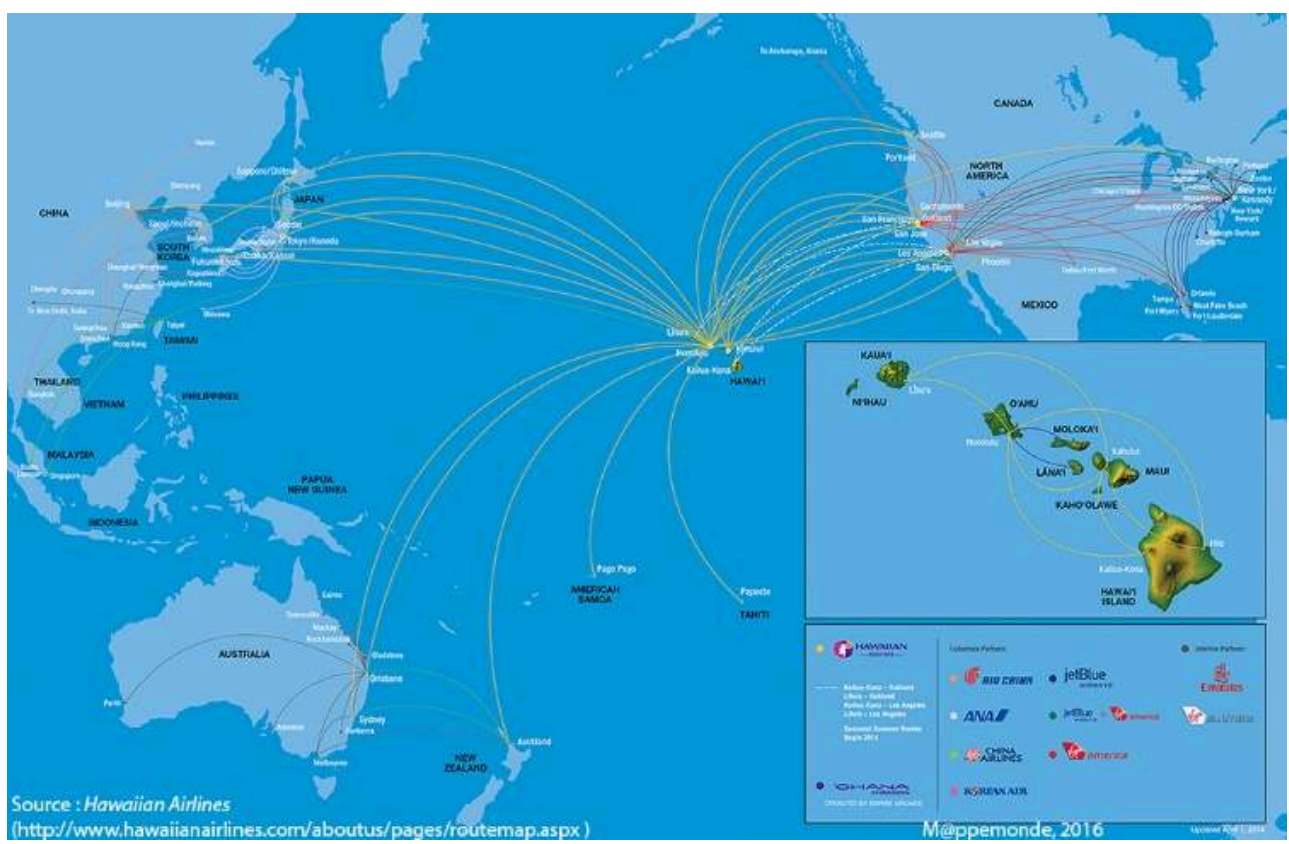

Source : Hawaiian Airlines, http://hawaiianairlines.com/aboutus/pages/routemap.aspx

On n'oubliera toutefois pas, en lisant ces cartes à petite échelle, que l'immensité demeure une réalité, comme le montre la carte du réseau d'Air Tahiti (figure 14): reporté sur une carte de l'Europe ses dessertes s'étendraient du nord au sud depuis Uppsala (Suède) jusqu'aux Pyrénées (ligne Nuku Hiva - Rimatara, îles Tubuai), d'ouest en est depuis Guernsey (îles anglo-normandes) jusqu'à Bucarest (ligne Maupiti Mangareva). Même avec la mondialisation et les progrès des techniques aéronautiques, la géographie - au sens le plus plat du terme - ne perd pas tout à fait ses droits. 


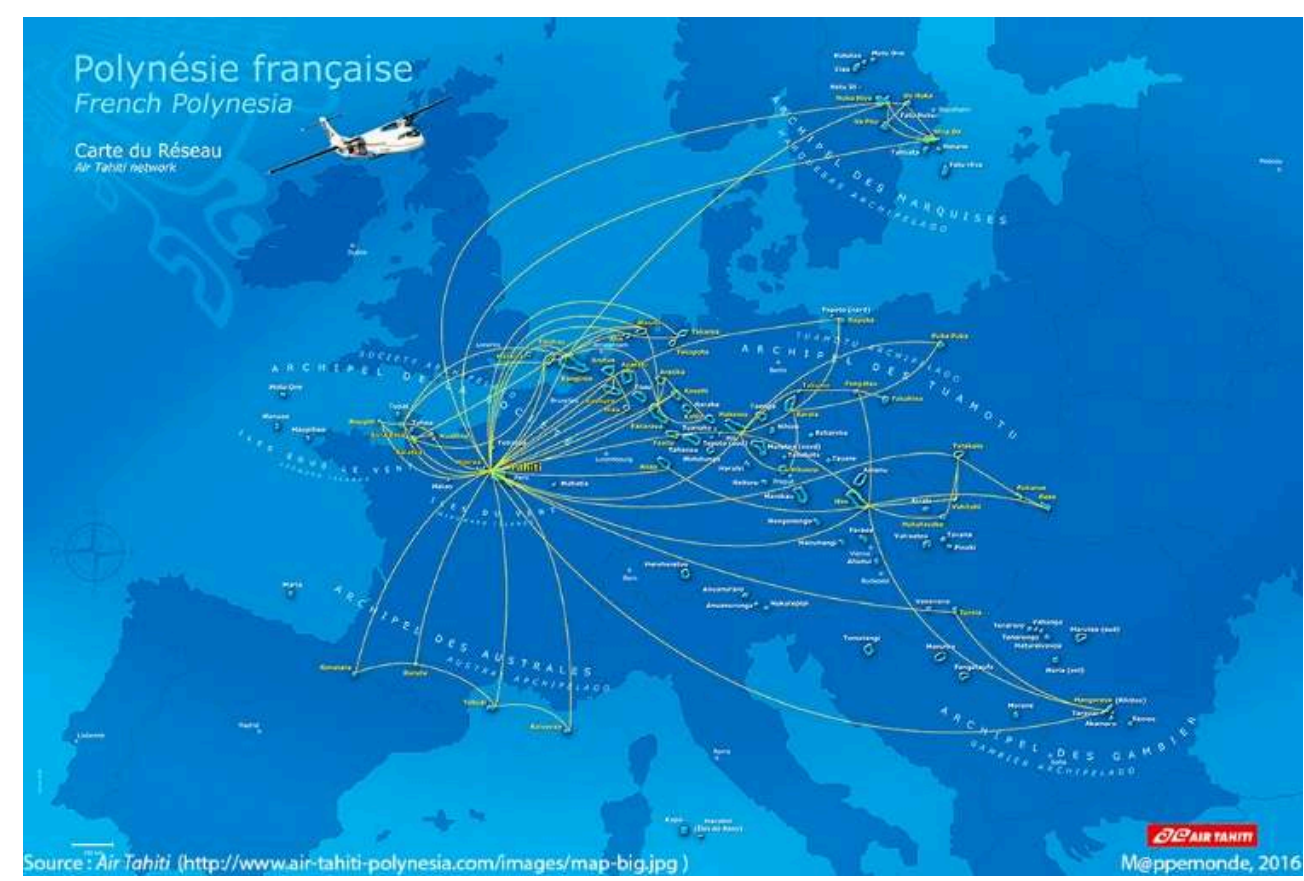

Source : Air Tahiti, http://www.air-tahiti-polynesia.com/images/map-big.jpg

Pour compléter cette analyse des cartes de réseaux il faudrait évidemment passer de 1 'échelle internationale à celle des pays, notamment des plus grands. Les sources utilisées jusqu'ici ne le permettent pas, il faut donc avoir recours à d'autres, ce qu'on peut faire sur un pays pour lequel d'abondantes données sont disponibles, le Brésil.

Il a été en 2014, selon la Banque Mondiale, au $8^{\mathrm{e}}$ rang mondial en nombre de passagers. La géométrie des lignes aériennes qui le relient au reste du monde est significative ${ }^{7}$ et en 2011, pour la première fois, le nombre de passagers voyageant en avion sur les lignes intérieures y a dépassé celui des voyageurs utilisant les bus interurbains ${ }^{8}$. Les transports aériens intérieurs y trouvent en efFet des conditions particulièrement favorables. D'un côté, la taille du pays (la $5^{\mathrm{e}}$ au monde) et la structure du peuplement en « archipel » créent une demande de déplacements à longue distance, de celles que l'avion franchit plus commodément que tout autre moyen de transport. D'un autre côté, la nécessité de gérer et d'administrer un espace très difFérencié suscite un important trafic d'afFaires, de fonctionnaires en déplacement et de politiciens en transit entre leurs circonscriptions et les capitales des États ou de l'Union. Et la concentration des revenus y assure qu'une partie au moins des 200 millions d'habitants du pays forment une clientèle solvable pour des déplacements privés ou de loisirs : plus de 10 millions de passagers par an empruntent les lignes intérieures. 
Figure 15. Trafic des aéroports et passagers au Brésil en 2010

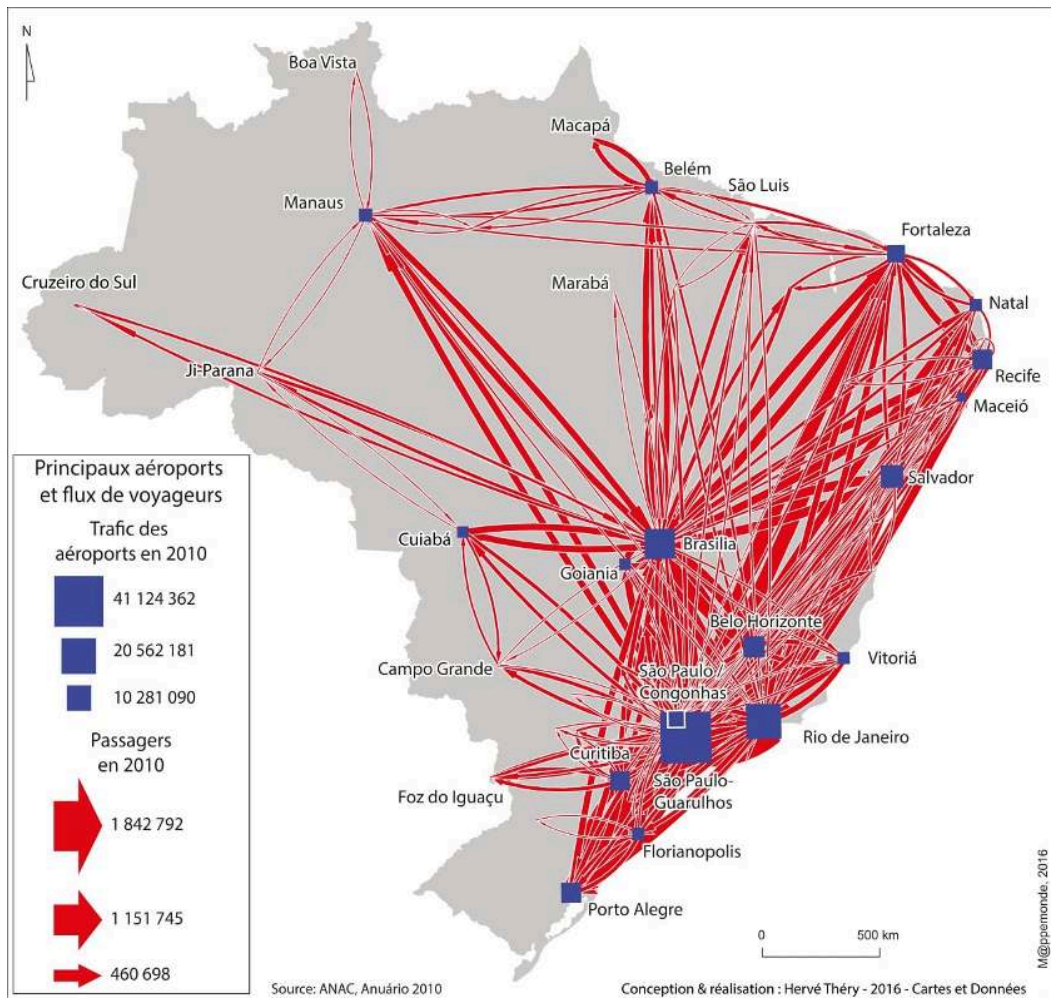

Conception et réalisation : Hervé Théry - 2016 - Cartes et Données

Source : ANAC, Anuário 2010

Grâce aux données publiées annuellement par l'ANAC (Agence nationale de l'aviation civile) on peut non seulement avoir une vision précise des lignes aériennes intérieures parcourues par toutes les compagnies, mais aussi de leur fréquentation. Les principaux flux accompagnent le littoral - la partie la plus densément peuplée du pays - de la frontière méridionale à la pointe du Nordeste, ou s'organisent autour de Brasília. La capitale du pays bénéficie en efFet de tout le trafic engendré par sa fonction politique (parlementaires du Sénat et la Chambre des Députés, fonctionnaires en mission, lobbyistes en tout genre), et par sa fonction internationale (ambassades, organisations et ONG internationales). Elle est en outre de plus en plus utilisée par les compagnies aériennes, en raison de sa position centrale, comme hub pour les relations entre les différentes régions du pays. 
Figure 16. Coût des voyages aériens au départ des principales villes brésiliennes

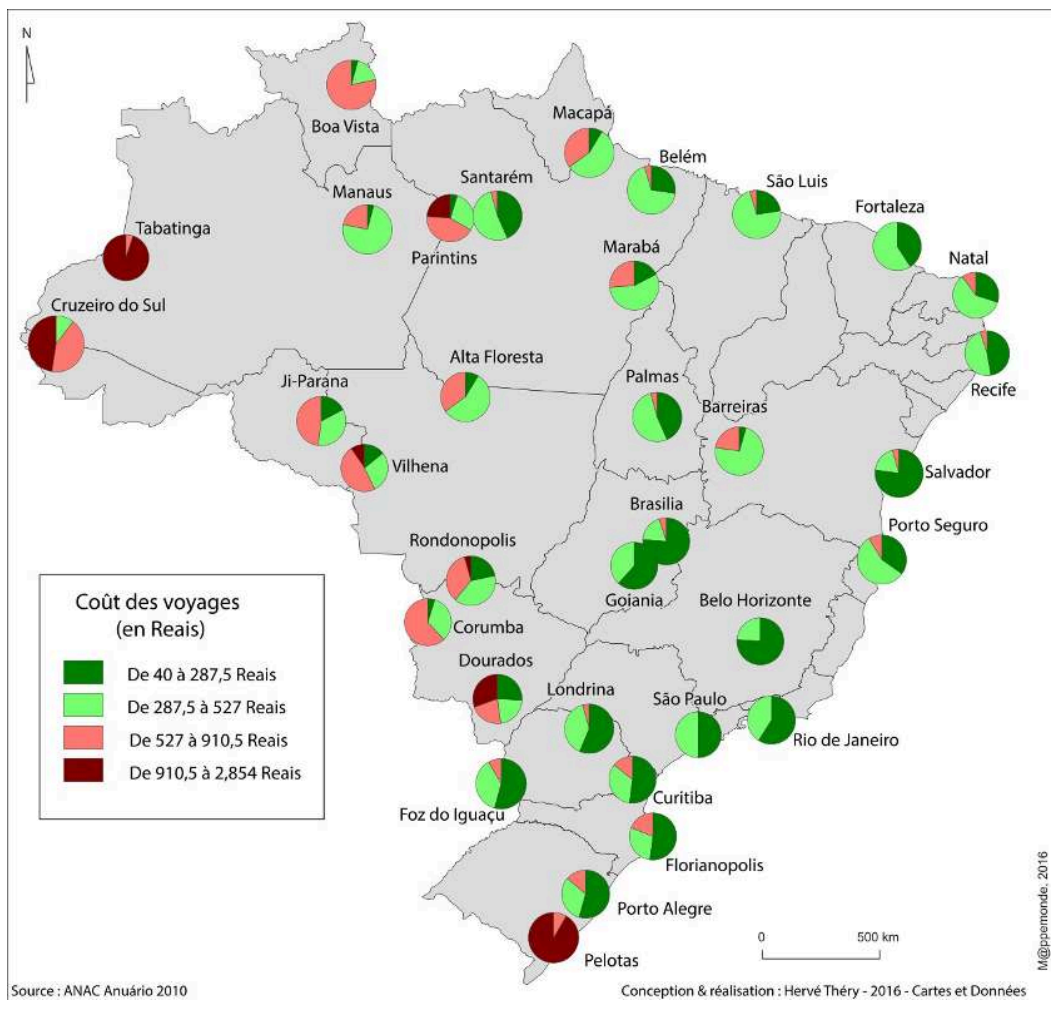

Conception et réalisation : Hervé Théry - 2016 - Cartes et Données

Source : ANAC, Anuário 2010

L'existence d'une ligne aérienne est toutefois une condition nécessaire mais non suffisante pour qu'un fort trafic s'y établisse, encore faut-il que ce soit à des prix acceptables pour la clientèle. Une carte élaborée à partir des coûts des billets montre un fort contraste entre les villes à partir desquelles les billets sont moins chers, dans les régions côtières les plus peuplées et sur l'axe Brasilia-Belém, et les plus chères dans l'ouest de l'Amazonie (surtout Tabatinga) ou l'extrême sud (surtout Pelotas).

Comme le montre cet exemple brésilien, changer d'échelle, disposer de statistiques de fréquentation des lignes et quantifier les coûts permet évidemment de tirer des analyses de réseaux aériens bien plus d'information sur les attracteurs et sur les flux qu'ils engendrent.

Mais, faute de pouvoir le faire autrement que pays par pays, les données tirées des transpondeurs des avions en vol, et plus encore des cartes fournies par les compagnies aériennes, présentent plusieurs avantages. Les premières sont gratuites et facilement accessibles sur internet, en quelques clics sur les principaux moteurs de recherche. Les secondes sont disponibles sur les sites des compagnies, qui les tiennent à jour et leur donnent un aspect aussi expressif et précis que possible pour attirer des clients sur leurs lignes plutôt que sur celles de leurs concurrents.

Leur motivation est donc purement - et légitimement - commerciale mais il est possible d'en faire un usage scientifique détourné, comme la rédaction de cet article, ou pour des usages pédagogiques. On imagine bien des élèves menant sur internet des recherches sur les lignes aériennes disponibles entre tel et tel pays, ou sur le faisceau de lignes de telle ou telle compagnie, etc. Parcourir le monde sur les ailes de 
l'imagination - et en tirer des leçons sur la géographie mondiale - est à portée de souris...

\section{BIBLIOGRAPHIE}

\section{Bibliographie}

Amiel M. MELANÇon G., ROZEnBlat C. (2005). « Réseaux Multi-Niveaux : L’exemple des échanges aériens mondiaux de passagers ». M@ppemonde, $\mathrm{n}^{\circ} 79$.

BIPLAN P. (2004). « Les compagnies aériennes entre la nation et la mondialisation ». Hérodote, $\mathrm{n}^{\circ} 114$, p. 57-70. DOI 10.3917/her.114.0056

CATTAN N. (2004). « Le monde au prisme des réseaux aériens ». Flux, 4/2004, n 58.

STRALE M. (2013). « Géographie mondiale des alliances de compagnies aériennes ». Belgeo , 4|2006.

THÉRY H. (2012). « Marine TraFFc project, un outil d'observation des routes et ports maritimes dans le monde ». M@ppemonde, $\mathrm{n}^{\circ} 104$.

VARLET J. (1997). « La déréglementation du transport aérien et ses conséquences sur les réseaux et sur les aéroports ». Annales de Géographie, 106 année, $n^{\circ}$ 593/594, Infrastructures de transports et organisation de l'espace en France au seuil du XXI ${ }^{e}$ siècle, p. 205-217. https://www.jstor.org/stable/ 23455101 ?seq=1

\section{Pour aller plus loin}

GRASLAND L., THERY H. (1994). « La généralisation des transports aériens en Europe : saturation du centre et redéploiement vers la périphérie ». M@ppemonde, 3-1994, p. 40-42.

IBGE (2013). Ligações Aéreas 2010 : conexão de seis cidades com São Paulo absorve $25 \%$ do transporte de passageiros. Censo 2010, Instituto Brasileiro de Geografia e Estatística.

PEREIRA A. P. C. (2014). Asas da centralidade em céus conhecidos : a dinâmica empresarial do setor de transporte aéreo no território brasileiro. Tese (Doutorado em Geografia Humana), Faculdade de Filosofia, Letras e Ciências Humanas, Universidade de São Paulo. https://teses.usp.br/teses/ disponiveis/8/8136/tde-06052015-145214/pt-br.php

THÉRY H. (2004). « Les transports aériens au Brésil, ou les ailes de la centralité », Problèmes d'Amérique latine, n 52, p. 85-98.

THERY H. (2015). « Representações de fluxos aéreos ». GEOUSP : Espaço e Tempo (Online), vol. 19, $\mathrm{n}^{\circ}$ 1, p. 160-165. http://www.revistas.usp.br/geousp/article/view/98642 


\section{NOTES}

1. Ces dispositifs, dont sont équipés tous les avions de ligne, transmettent constamment leur position aux centres de contrôle aérien pour éviter les collisions.

2. J'avoue l'avoir fait parfois, avec un sentiment de culpabilité - ça ne se fait pas d'arracher les pages des livres, ni même des revues - atténuée par le fait que ces revues sont offertes aux passagers et que j'aurais pu les emporter à la fin du voyage. Mais seule cette page m'intéressait...

3. De la même façon que le Marine TrafFc project, un excellent outil d'observation des routes et ports maritimes dans le monde, déjà présenté dans M@ppemonde (Théry 2012).

4. Notamment https://www.youtube.com/watch?v=_NFGD9cglb4 ou https:// www.youtube.com/watch ?v=LrxalYXXBfI. Pour un zoom sur l'Europe https:// www.youtube.com/watch ?v =BI4jrAq6idI ou https://www.youtube.com/watch ?v =s2b06qtqpp 5. http://ec.europa.eu/transport/modes/air/safety/air-ban/index_fr.htm 2 http:// www.developpement-durable.gouv.fr/Liste-noire-des-compagnies,27632.html

6. Transportes aéreos portugueses, récemment achetés par le propriétaire de la compagnie brésilienne Azul, associé à un investisseur portugais.

7. Carnet de recherche Braises, «La place du Brésil dans le monde mesurée par les transports aériens et le commerce international »

8. Carnet de recherche Braises : «Avions vs bus »

\section{RÉSUMÉS}

Le transport aérien est sans aucun doute l'un des éléments majeurs de la mondialisation. Tout comme elle, il a sa géographie, faite de points-clés - les aéroports plus ou moins fréquentés - et ses lignes de force, les lignes aériennes. L'article analyse cette géographie, en se fondant principalement sur l'analyse de cartes de réseaux, en passant du niveau global à celui des alliances et des compagnies, et montre les efFets d'un mouvement de conquête des réseaux mondiaux par certains pays émergents.

Air transportation is unequivocally a major element of globalization. As with globalization, it has its own geography, made of key points (airports with varying degrees of traffic) and lines of force (airlines). This article analyzes its geography using the analysis of network maps, from the global level to that of alliances and companies. It also shows how certain emerging countries are conquering global networks.

El transporte aéreo es, sin duda alguna, uno de los factores clave de la globalización. Del mismo modo que ésta tiene una geografía con centros estratégicos y espacios de referencia, los aeropuertos también la tienen con sus frecuencias, ocupación, actividad, enlaces y compañías aéreas. Mediante la cartografía de flujos, el artículo analiza desde la generalidad de las redes a la particularidad de las alianzas entre compañías y empresas. Además, muestra la paulatina conquista de estas redes mundiales por algunos países emergentes. 
INDEX

Keywords : airlines, alliances, globalization, emerging countries

Palabras claves : aerolíneas, alianzas, globalización, países emergentes

Mots-clés : alliances, globalisation, lignes aériennes, pays émergents

\section{AUTEUR}

\section{HERVÉ THÉRY}

Directeur de recherche émérite au CNRS. Professor visitante na Universidade de Sao Paulo (USP) Co-directeur de la revue Confins (http://journals.openedition.org/confins) Blog de recherche Braises (http://braises.hypotheses.org) 